\title{
Transforaminal Endoscopic Surgery: Its Role in the Treatment of Painful Conditions of an Aging Spine
}

\section{Anthony T Yeung*}

University of New Mexico, School of Medicine, Albuquerque, New Mexico, USA

*Corresponding author: Anthony T Yeung, University of New Mexico, School of Medicine, Albuquerque, New Mexico, USA, Tel: (602) 944-2900; E-mail: ayeung@sciatica.com

Rec date: Sep 18, 2016; Acc date: Sep 20, 2016; Pub date: Sep 24, 2016

Copyright: (C) 2016 Yeung AT. This is an open-access article distributed under the terms of the Creative Commons Attribution License, which permits unrestricted use, distribution, and reproduction in any medium, provided the original author and source are credited.

\section{Opinion}

A major gap exists between fusion oriented surgeons and Pain Management leaders, bridged by transforaminal endoscopic surgeons represented by the International Intradiscal and Transforaminal Therapy Society (formerly IITS). Current surgical philosophy by key opinion leaders (KOL's) focus on attaining fusion of the painful spinal segment by emphasizing femoral-pelvic and overall spine sagittal alignment, representing another phase in the evolution of fusion techniques. For pain management, Neuro Modulation of the Spinal Cord and more recently, the Dorsal Root Ganglion is the area of focus as represented by the key opinion leaders, Gabor Racz and the World Institute of Pain (WIP). Endoscopic surgeons support "full endoscopic" decompression procedures as the least invasive of the trend toward minimally invasiveness in spine surgery. Notably, transforaminal decompression is still under-valued and under-utilized because formal training in academic centers is lacking, and since advancements have been made in private practice settings.

The current health care environment in the USA places emphasis on stemming the escalating cost of spine care by government and insurance payers, yet fails to give priority to what is medically best for the patient. Payment is increasingly governed by artificial guidelines developed by each payer with emphasis on evidenced based guidelines (EBM) that are more focused on cost management than efficacy and safety. With denial of procedures requested by surgical and nonsurgical interventionalists, providers' medical decisions become more dictated by codes, re-imbursement, and playing the re-imbursement game than on the patient. An opioid crisis has therefore developed in the USA, when practioners find the least path of resistance is to prescribe painkillers.

All competing factions need new directions, with cooperation between physicians and other health care providers to be aware of each other's contributions, and to coordinate care with cooperation, or there will be a need for competing providers to warrantee their procedures much like consumer products are warranted.

The individual needs of our patients are too restricted by the use of artificial guidelines that restrict payment and physicians' autonomy to treat our patients for their individual needs. By focusing on the pathoanatomic source of pain by endoscopic visualization, surgical pain management of pain is a viable treatment option validated in peer reviewed publications. I refer the reader to my last publication in Surgical Technology International, "In-vivo endoscopic visualization of patho-anatomy in symptomatic degenerative conditions of the lumbar spine II: Intradiscal, foraminal, and central canal decompression." Surg Technol Int. 2011 Dec 1; XXI: 299-319 for background information that will serve as a reference for this monologue of my personal experience in this evolving field.
The treatment algorhythim in spine is evolving and changing due to a better understanding of the patho-anatomy, well correlated with its pathophysiology. I have published my evolving technique in past publications, most in a series of articles in Surgical Technology International, beginning with the VIII edition and followed by the XI, $\mathrm{XV}$ and XXI edition.

Pain is better understood with in vivo visualization and probing of the pain generators using endoscopic transforaminal access rather than just relying on a symptom diagram and image correlation (1). This culminates in a shared decision making process involving patient and surgeon, focused on a broader spectrum of surgical as well as nonsurgical treatments, and not just masking the pain generator. Transforaminal endoscopic decompression, irrigation, and ablation have moved away from decisions based on just imaging confirmation alone. Image abnormalities alone cannot explain the pain and disability experienced by each individual patient. Images do not always show variations in nerve supply and patho-anatomy, or do they quantify the pain experienced by each individual patient. The ability to isolate and visualize "pain" generators in the foramen and treating persistent pain by visualizing inflammation and compression of nerves, serves as the basis for transforaminal endoscopic [TFE] surgery [1,2]. This has also resulted in better pre- surgical planning with more specific and defined goals in mind. There are various surgical philosophies and techniques proposed by other pioneers in endoscopic surgery, but I choose to embrace the "inside out" philosophy of TFE surgery as safe and precise in trained and good surgical hands. It provides basic access to the disc and foramen that cover a large spectrum of painful pathologies with the least surgical risk.

I begin with a personal account of my own spinal condition that deteriorated rapidly over the past 5 years. My focus on treating pain generators with transforaminal endscopic surgery over the past 25 years caused me to have the surgery I helped developed on myself, performed by my son, Chris Yeung, M.D., an associate at Desert Institute for Spine Care, our group practice.

In 1991, I took Parviz Kambin's course in arthroscopic spine surgery and, with extensive personal experience in joint arthroscopy, began using the endoscope to explore intradiscal and foraminal anatomy. I studied Rauschning's cadaver cryo-anatomy. It seemed natural to me to utilize the endoscope to examine patho-anatomy in the spine. I quickly decided to also visualize the epidural space through the foramen with the "inside out" technique to obtain confirmation that the spinal nerves were decompressed. Also, in 1991, the laser was introduced for the lumbar spine, and I became a fan of laser as a surgical tool under endoscopic visualization because it had the tissue effect of decreasing bleeding and ablating soft tissue and bone through a $6 \mathrm{~mm}$ working cannula with a $2.8 \mathrm{~mm}$ working channel scope. My experience caused me to develop my own endoscopic system in conjunction with Richard 
Citation: Yeung AT (2016) Transforaminal Endoscopic Surgery: Its Role in the Treatment of Painful Conditions of an Aging Spine. J Neurol

Wolf Surgical Instrument system, and marketed the FDA approved system in 1997, called the Yeung endoscopic spine system (YESS ${ }^{\mathrm{m}}$ ) (Figure 1).

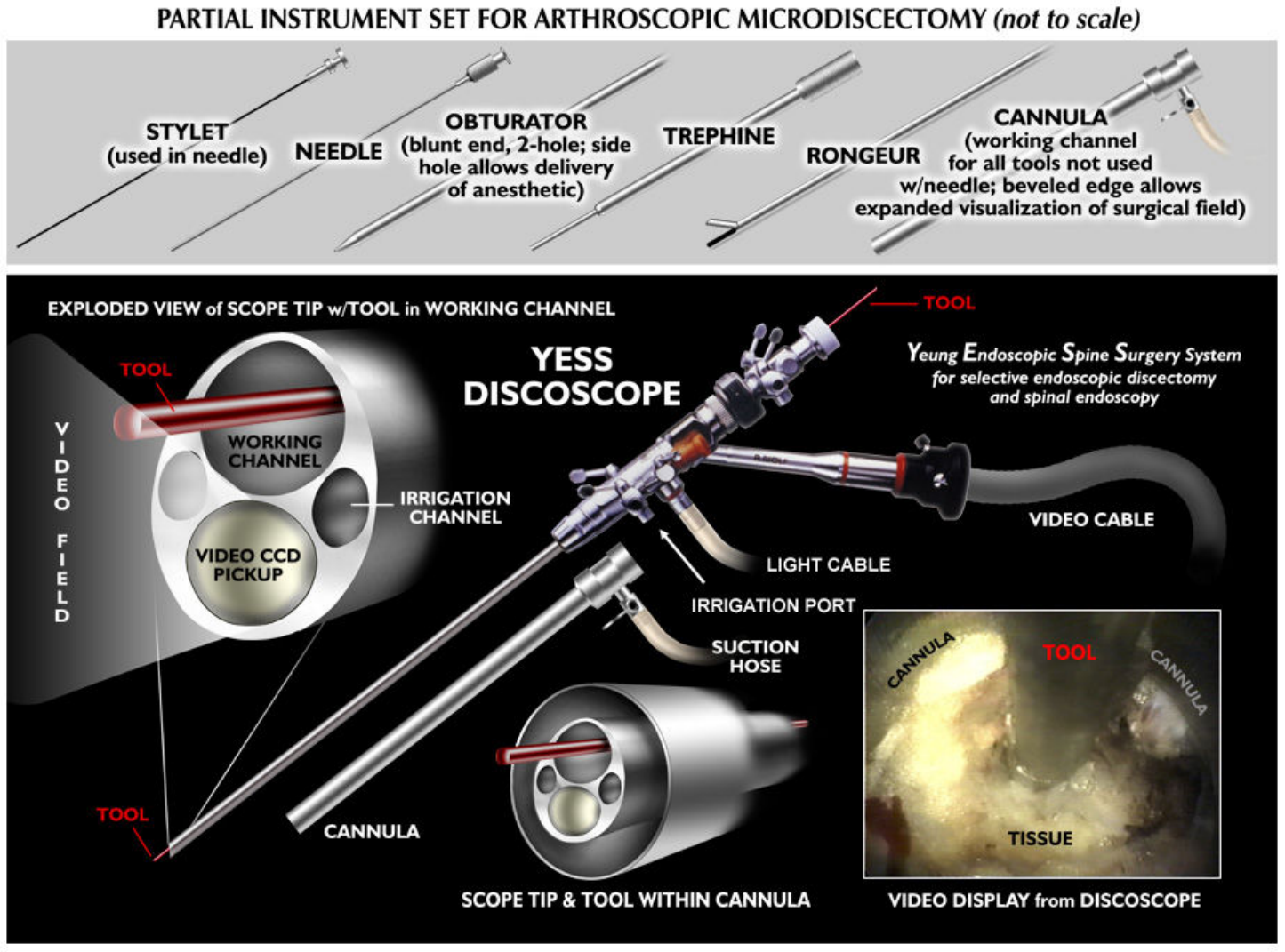

Figure 1: YESS endoscope.

I have not deviated from this surgical philosophy since then, except to constantly evolve the technique, aided by my recognition of spinal patho-anatomy. I customized surgical instrumentation that was developed to facilitate decompression, ablation, and irrigation of the pain generator. I documented patho-anatomy identified by the endoscope and monitored the effect by probing, decompression, ablation or transection. The original endoscope design is still the state of the art configuration for intradiscal therapy, where the degenerative process begins. It has been copied to some extent in the endoscope designs of competing endoscope companies. As the procedure evolved, different scope sizes, designs and working channels were augmented by better endoscopic instrumentation and modified approach techniques. The instruments have evolved to make endoscopic spine surgery a staple of surgical treatment of the future. With the current interest in biologics, we are destined to improve spine care by moving away from fusion as a first line surgical treatment because of the techniques available in the endoscopic platform.

\section{References}

1. Yeung AT, Gore SR (2001) Evolving methodology in treating discogenic back pain by Selective Endoscopic Discectomy (SED) and thermal annuloplasty. Journal of Minimally Invasive Spinal Techniques 1: 8-16.

2. Gore SR, Yeung AT (2003) Identifying sources of discogenic pain. Journal of Minimally Invasive Spinal Techniques 3: 21-24. 